\title{
THE EVOLUTIONARY STATUS OF PSR 1718-19
}

\author{
ENE ERGMA \\ Physics Department, Tartu University, Ulikooli 18, \\ EE2400 Tartu, Estonia.
}

\author{
AND \\ MAREK J. SARNA \\ N. Copernicus Astronomical Center, \\ Polish Academy of Sciences, ul. Bartycka 18, 00-716 Warsaw, \\ Poland.
}

\begin{abstract}
Possible models for the matter source inside the eclipsing binary system PSR 1718-19, and for the evolution of this system are reviewed, including Zwitter's (1993) stripped main-sequence (MS) turnoff star model. Both the accretion induced collapse (AIC) scenario with a young neutron star, and the capture scenario with an old neutron star are discussed. Although Burderi \& King (1994) claim that the size of the Roche lobe $\left(\sim 0.5 \mathrm{R}_{\odot}\right)$ unambiguously rules out the AIC formation scenario, we show that in our evolutionary picture an AIC scenario will be possible.
\end{abstract}

\section{Introduction}

Lyne et al. (1993) discovered the binary radio pulsar PSR 1718-19 in the globular cluster NGC 6342. It is a 6.2 hour eclipsing binary system; the pulsar has a relatively long spin period of $1 \mathrm{~s}$, a very strong magnetic field $\left(\sim 10^{12} \mathrm{G}\right)$, and a small spin down age $(\sim 10 \mathrm{Myr})$. The mass of its companion is only $0.1-0.2 \mathrm{M}_{\odot}$. The discovery of this system increases the number of systems in which the neutron star companion mass is low. Lyne et al. pointed out that there is a problem in explaining the source of material in this eclipsing system. They showed that for a MS star with $M_{\mathrm{c}} \sim 0.1-$ $0.2 \mathrm{M}_{\odot}$ the surface of the companion is well inside its Roche lobe. Also the possibility that the eclipsing material results from the ablation of the surface of the companion star by radiation from the pulsar, derived from its 
spin-down energy, fails. Due to the large spin period of the neutron star the rotational energy flux at the companion is too small $\left(\sim 210^{9} \mathrm{erg} \mathrm{s}^{-1} \mathrm{~cm}^{-2}\right)$ to cause significant evaporative mass loss.

The question arises whether this system is associated with the globular cluster NCG 6342. It lies at about 2.3 arcmin (or 17 core radii) from its center. Lyne et al. (1993) have argued that the position, distance and binary nature suggest that the system is probably associated with the cluster, although there is small $(<1 \%)$ but not negligible probability that its association with the cluster is a chance superposition (Wijers \& Paczynski 1993).

If we accept that the binary is a globular-cluster member then we need an extra mechanism to bring it to the outskirts of the cluster: in the old neutron star capture scenario the binary must have originated in the core of the cluster.

Another important question is whether the pulsar has been recently formed ( $\sim 10^{7}$ yrs ago), or is an old object. Unfortunately, it seems to us that this question can not be easily answered.

So far, several models have been proposed to explain the source of the matter in this eclipsing binary system. We shall give a brief review of these models. Then, using the model of Zwitter (1993), we describe evolutionary scenarios including both the young neutron star version as well as the capture version (old neutron star).

\section{How to explain Matter inside the Binary System?}

Ergma (1993) invoked X-ray pre-heating of the secondary in AM Her type binaries. She showed that if the hard X-ray luminosity is of the order of $10^{33} \mathrm{erg} \mathrm{s}^{-1}$ then the secondary will be heated and be out of thermal equilibrium. After collapse of the massive white dwarf, and spin down of the neutron star to its current rotational period the pulsar action will switch off. Since the thermal time scale of the companion is much longer $(\sim 100 \mathrm{Myr})$ than the system time scale $(\sim 10 \mathrm{Myr})$ the companion has no time to relax to its thermal equilibrium, and its radius will be larger than its equilibrium value. It may nearly fill its Roche lobe and hence be a source of the material inside the system.

Zwitter (1993) has argued that the companion is the stripped turn off star of $0.2-0.4 \mathrm{M}_{\odot}$ with an $\sim 0.1 \mathrm{M}_{\odot}$ helium core, and an $\sim 1.8$ times bigger radius than a MS star of same mass. Its position in the H-R diagram overlaps that of an $\sim 0.65 \mathrm{M}_{\odot}$ MS star, and it is then only slightly underfilling its Roche lobe near $P=6.2 \mathrm{hrs}$.

Wijers \& Paczynski (1993) have discussed the possibility that this system is old, and has been formed via capture of the companion by an old 
neutron star. To explain the matter inside the system they proposed that the companion is similar to the very active stars found in RS CVn systems. The cause of mass loss in these binaries is thought to be the combination of magnetic activity and the rapid rotation.

To explain the low-mass companion for this system, Verbunt (1994) proposed a new mechanism that leads to such low companion masses extreme tidal heating as the orbit circularizes when the neutron star is formed, or is exchanged into the binary. The circularization is accompanied by heating of the companion of the neutron star and the amount of energy produced can be comparable to the binding energy of the companion which may cause the strong mass loss. Thus the star will have again a radius larger than a MS star of the same mass.

So three models (Ergma, Zwitter, Verbunt) predict that the secondary is out of thermal equilibrium and hence must be rather bright.

Wijers \& Paczynski (1993) have proposed an observational test to clarify the nature of the secondary [in their model neither a normal low-mass star nor a stripped (bloated) star]. If the optical counterpart is a MS star then it should have $m_{\mathrm{I}}>24.5$ and $R-I>1$.1. If the pulsar formed via collapse of a white dwarf or has a stripped companion star then $m_{\mathrm{I}} \sim 22.6$ and $R-I \sim$ 0.5 . To detect the companion a more accurate position determination is needed, which is not yet available (Lorimer, private communication).

\section{Scenario with a Young Pulsar}

Let us assume an initial system with a massive $\mathrm{NeOMg}$ white dwarf and with slightly evolved secondary. For this case initial Roche lobe filling will occur at a relatively large orbital period $(P \sim 19 \mathrm{hr})$, when the secondary will have a small helium core $\left(M_{\mathrm{He}} \sim 0.01 \mathrm{M}_{\odot}\right)$. The mass transfer starts and the system evolves, driven by magnetic braking. Near an orbital period of $6 \mathrm{hr}$ the mass of the secondary has decreased to $0.2 \mathrm{M}_{\odot}$ (Fig. 1). Let us suppose that the accretion induced collapse happened near an orbital period of $\sim 6.1 \mathrm{hr}$. The maximum value of the mass lost in the form of binding energy is roughly $0.18 \mathrm{M}_{\odot}$ (Zeldovich \& Novikov 1971), but recent computations of accretion induced collapse of a massive $\mathrm{NeOMg}$ white dwarf $\left(M_{\mathrm{NeOMg}}=1.376 \mathrm{M}_{\odot}\right)$ by Woosley \& Baron $(1992)$ have shown that only about $\sim 0.01 \mathrm{M}_{\odot}$ has been ejected.

If we accept that there is no other "internal" kick mechanism operating, this mass loss value will lead to the following post-collapse eccentricity, semi-major axis and centre of mass velocity of the system (Dewey \& Cordes 1987):

$$
e=\frac{\Delta M}{M_{1}+M_{2}-\Delta M}, \quad a_{\mathrm{f}}=a_{\mathrm{i}} /(1-e), \quad v_{\mathrm{s}}=e v_{1}
$$




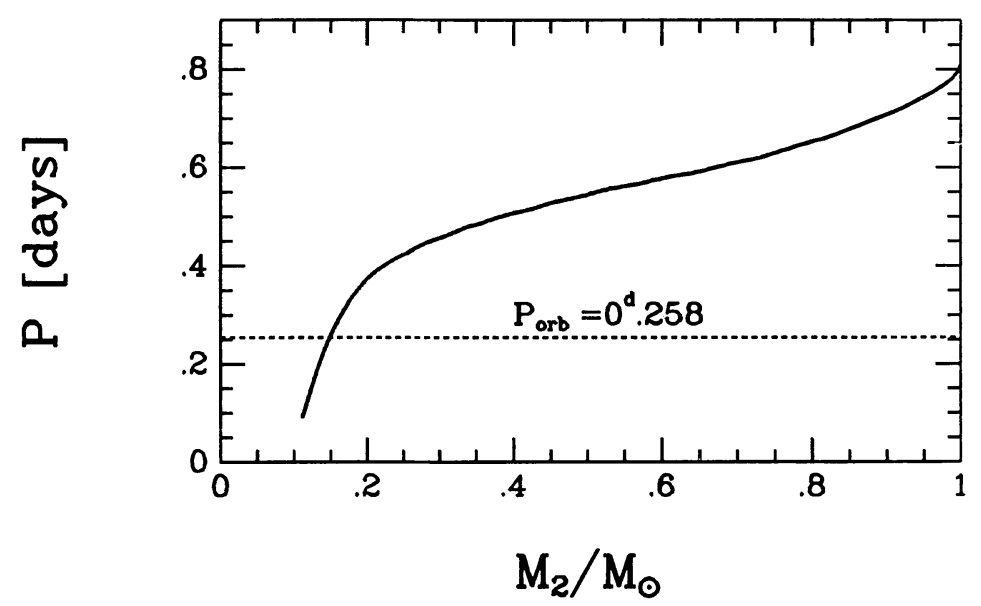

Figure 1. The secondary mass and orbital-period dependence.

For $M_{1}=1.376 \mathrm{M}_{\odot}, M_{2} \sim 0.2 \mathrm{M}_{\odot}, \Delta M \sim 0.01 \mathrm{M}_{\odot}$, we have $e \sim 610^{-3}$. Although the post-collapse orbital separation differs very little from the pre-collapse orbital separation it may be enough to force the secondary to be slightly underfilling its Roche lobe. Since pre- and post-collapse orbital separations are very similar, no violent tidal circularization of the orbit will occur, as it was suggested for large eccentricities by Verbunt (1994), The pre-collapse orbital velocity $v_{1}$ (for $P \sim 6.1 \mathrm{hr}$ ) is $\sim 50 \mathrm{~km} \mathrm{~s}^{-1}$, hence the post-collapse centre of mass velocity is $\sim 0.3 \mathrm{~km} \mathrm{~s}^{-1}$. This value is less than the central velocity dispersion of NGC $6342\left(v_{\mathrm{c}}=8 \mathrm{~km} \mathrm{~s}^{-1}\right.$, Pryor \& Meylan 1993), so it is insufficient to move the binary outside the center of cluster.

\subsection{AIC AND NOVA EXPLOSION}

The AIC scenario requires that there must be cataclysmic variables in globular clusters. Up to now, CVs are the rarest type of binaries known in globular clusters (see, e.g., Shara et al. 1994). Also, theoretical views on this topic are quite controversial. While Hertz \& Grindlay (1983) and Rappaport \& Di Stefano (1993) have suggested high numbers of CVs in globular clusters, Verbunt \& Meylan (1988) predicted a much lower number on the basis of the lower capture probability of WDs compared to NS.

If we assume a normal $\mathrm{CV}$ evolution in globular clusters then we have to face a second problem. It is connected with nova explosions and the subsequent mass loss, which does not allow the increase of the mass of the white dwarf required for AIC. Truran et al. (1988) have shown that if the accreted matter is hydrogen poor (the hydrogen content by mass is less 


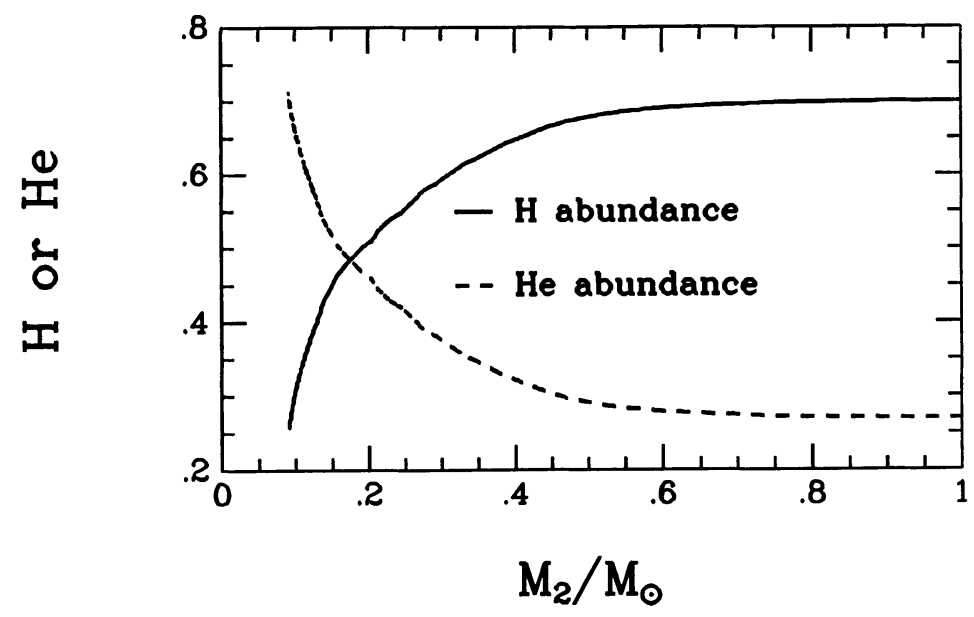

Figure 2. The hydrogen (thick line) and helium content (dashed line) dependence on the secondary mass; it is assumed that $M_{1}=1.4 \mathrm{M}_{\odot}$.

than 0.6) then significant radius expansion does not occur and no mass is ejected during a thermonuclear flash. According to our calculations (Fig. 2) the surface hydrogen abundance is less than 0.6 when the secondary mass has decreased to $0.36 \mathrm{M}_{\odot}$. The it is possible to accrete $\sim 0.16 \mathrm{M}_{\odot}$ without mass ejection, and hence the AIC may occur (see also Livio 1992). We shall present more details in our full paper (Ergma et al. 1994).

\section{Old Neutron Star Scenario}

In this scenario the binary has originated at the center of the cluster by the tidal capture of the secondary by an old neutron star.

For the companion to be captured but to have avoided destruction, its distance of closest approach to the neutron star at the first passage, $d$, must have been close to $3 R_{c}$. If we assume conservation of angular momentum during the circularization the final distance is $a_{\mathrm{f}}=2 d=6 \mathrm{R}_{\mathrm{c}}$ (Wijers \& Paczynski 1993). As an estimate we take $a_{\mathrm{f}}=(4-6) \mathrm{R}_{\mathrm{c}}$. We also propose that the normal companion with mass about $1 \mathrm{M}_{\odot}$ is a slightly evolved star (as is the case with the young neutron star version). If the $1 \mathrm{M}_{\odot}$ star has an age of $\sim 10^{10} \mathrm{yrs}$ its radius is $1.36 \mathrm{R}_{\odot}$. After circularization the final orbital separation $a_{\mathrm{f}}=(5.44-8.16) \mathrm{R}_{\odot}$ and the orbital period $P_{\mathrm{f}}=0.95-1.75$ days. Magnetic braking will decrease the orbital separation, and the star with $R_{\mathrm{c}}=1.36 \mathrm{R}_{\odot}$ will fill its Roche lobe when $P=0.57 \mathrm{~d}$. To decrease the period from $P_{\mathrm{f}}$ to $P=0.57 \mathrm{~d}$ a time interval of $\sim 10^{8} \mathrm{yr}$ is needed. After filling the Roche lobe the evolutionary path for this case is similar to that in the AIC scenario (Fig. 1). Only, we ought to follow also the history of the 
neutron star and of its field in a manner described by Muslimov \& Sarna (1993). Since we have assumed that the captured neutron star was old its initial rotational period (before the capture) may have been of the order of several seconds. After starting the mass transfer the pulsar rotational period decreases. When the orbital period is about 6-7 hours a third body is assumed to arrive near the binary (the pulsar period is then $\sim 1 \mathrm{~s}$ ). The whole system receives some additional kick velocity which may bring the binary to the outskirts of the cluster (Hills 1976). If the binary was ejected from the center of the cluster due to this three-body interaction then its space velocity must be between $v_{\mathrm{c}}$ and $v_{\mathrm{esc}}=2 v_{\mathrm{c}}$. We can see that the time required to bring the binary to its current place is (3-6) $10^{5} \mathrm{yrs}$ which is much less than the system life time estimated from $\dot{P}$ and $P$ (Lyne et al. 1993).

\section{The Fate of the System in both Scenarios}

Since the Roche lobe filling star originally was evolved, its future evolution will be as follows. According to Tutukov et al. (1985) (see also Ergma 1991) the star becomes helium rich during the mass loss and it will evolve towards very short orbital periods.

If magnetic-field decay occurs it is possible to predict an evolutionary sequence such as PSR 1718-19 $\Longrightarrow$ PSR 1744-24A $\Longrightarrow$ X 1916-05 $\Longrightarrow$ $X 1820-30$. If there is no magnetic field decay then we may expect that PSR 1718-19 evolves to a low-mass X-ray binary pulsar with a very short orbital period, like $\mathrm{X}$ 1627-67 (Levine et al. 1988).

\section{Discussion}

In their recent paper Burderi \& King (1994) claim that the Roche lobe radius value $\left(\sim 0.5 \mathrm{R}_{\odot}\right)$ unambiguously rules out the AIC formation scenario which requires a lobe-filling companion close to the main sequence. In our evolutionary picture we have shown that it is not necessarily the case. Having a slightly evolved star as the secondary at the beginning of the binary evolution, the observed secondary mass as well as the near filling of the Roche lobe are easily achieved. We think that the observational test which has been proposed by Wijers \& Paczynski (1993) is very important to restrict the proposed models.

Acknowledgments. This work was supported in part by the Polish National Committee for Scientific Research grant and the Estonian Science Foundation grant N 625. Part of this paper has been prepared during the visit of $\mathrm{E}^{2}$ to NORDITA, whose financial aid is acknowledged. 


\section{References}

Burderi, L. \& King, A.R. 1994, ApJ 430, L57

Dewey, R.J. \& Cordes, J.M. 1987, ApJ 321, 780

Ergma, E. 1991, Comments on Astrophys. 15, 239

Ergma, E. 1993, A\&A 273, L38

Ergma, E., Sarna, M.J. \& Giersz, M. 1994, (in preparation)

Hertz, P. \& Grindlay, J. 1983, ApJ 273, 105

Hills, J. 1976, MNRAS 175, 1P

Levine, A. et al. 1988, ApJ 327, 732

Livio, M. 1992, in Interacting Binaries, H. Nussbaumer \& A. Orr (Eds.), Springer Verlag (Berlin), p. 250

Lyne, A., Biggs, J., Harrison, P. \& Bailes, M. 1993, Nat 361, 47

Muslimov, A.G. \& Sarna, M.J. 1993, MNRAS 262, 164

Pryor, C. \& Meylan, G. 1993, ESO preprint No. 932

Rappaport, S. \& Di Stefano, R. 1993, in Cataclysmic Variables and Related Physics, 2nd Technion Haifa Conf., O. Regev \& G. Shaviv (Eds.), Israel Phys. Soc. (Jerusalem), p. 48

Shara, M.M., Bergeson, L.E. \& Moffat, A.F.J. 1994, ApJ 429, 767

Truran, J.W. et al. 1988, ApJ 324, 345

Tutukov, A.V. et al. 1985, SvAL 11, 52

Verbunt, F. 1994, A\&A 285, L21

Verbunt, F. \& Meylan, G. 1988, A\&A 203, 297

Wijers, R. \& Paczynski, B. 1993, ApJ 415, L115

Woosley, S.E. \& Baron, E. 1992, ApJ 391, 228

Zeldovich, Ya. \& Novikov, I.D. 1971, Relativistic Astrophysics, Vol. 1, Stars and Relativity, University of Chicago Press

Zwitter, T. 1993, MNRAS 264, L3

\section{Discussion}

S.R. Kulkarni: The location (140 arcsec from the core) of PSR 1718-19 is puzzling. Compare this with the half mass radius, i.e., $\sim 40$ arcsec. This means one has to finetune the systemic velocity in order not to lose the pulsar (to the Galactic field). I wonder how improbable this event is, from a dynamical point of view.

H. Ritter: First, I would like to disagree with the comment made by $\mathrm{dr}$ Lipunov, namely that white dwarfs, if sufficiently magnetic, can grow in mass even at low accretion rates. After all, nova Cyg 1975 is an AM Her star, i.e., has a strongly magnetic white dwarf; yet it underwent a nova explosion and ejected mass. Second, as to Nomoto's results about core collapse of an accreting white dwarf at $\dot{M} \approx 10^{-9} \mathrm{M}_{\odot} \mathrm{yr}^{-1}$, this accretion rate refers to accretion of helium, but not of hydrogen-rich matter. 